\title{
Estudio de la depresión en estudiantes de la Ciudad de México y del Estado de Michoacán por medio de la versión revisada de la CES-D
}

\author{
Alberto Jiménez Tapia, ${ }^{1}$ Fernando Wagner, ${ }^{2}$ María Elena Rivera Heredia, ${ }^{3}$ Catalina González-Forteza ${ }^{1}$
}

Artículo original

\section{ABSTRACT}

\section{Background}

The prevalence of depression is increasing among adolescents. Depression is related to factors such as socioeconomic status, family history of problems with depression and alcohol use, experiences with violence, physical or sexual abuse and use of tobacco and illicit drugs. These may increase the risk of other problematic behaviors.

\section{Objective}

To describe the depressive symptoms in adolescents from Mexico City and the State of Michoacan.

\section{Method}

Data were generated with the revised version of the CES-D. Two cross-sectional studies with non-probabilistic samples were conducted $(\mathrm{N}=2127)$.

\section{Results}

A total of $12 \%$ of the adolescents had symptoms of a probable major depressive episode (MDE) (13.3\% from Mexico City and 9.2\% from Michoacan). The proportion of probable MDE was significantly higher among women $\left(\chi^{2}=56.294, \mathrm{DF}=2, p<.001\right)$. The students from Mexico City had a significantly higher proportion of subjects with probable $M D E$ than the ones from Michoacan $\left(\chi^{2}=30.78, D F=2, p<.001\right)$.

\section{Discussion and conclusion}

The proportion of students who had clinically significant symptoms underlines the need for information, awareness, training for parents, teachers, health professionals and adolescents for addressing the relevance for attending depression and to improve the access to health care services.

Although the CESD-R may be a quick alternative for an early detection of a probable major depressive episode, it would be necessary to build a referral mechanism to mental health care services for individuals at risk, as well as strategies to ensure its quality.

Key words: Adolescents, students, depression, CESD-R.

\section{RESUMEN}

\section{Antecedentes}

La prevalencia de la depresión es cada vez mayor en los adolescentes y se relaciona con factores como el nivel socioeconómico, la historia familiar con problemas de depresión y de consumo de alcohol, experiencias con la violencia, abuso físico o sexual, así como consumo de tabaco y de drogas ilegales, todas las cuales aumentan el riesgo de otras conductas problemáticas.

\section{Objetivo}

Describir los síntomas depresivos en adolescentes de la Ciudad de México y del Estado de Michoacán.

\section{Método}

Se hicieron dos estudios transversales con muestras no probabilísticas ( $N=2127$ ), utilizando las categorías clínicas de la Escala CESD-R.

\section{Resultados}

Un $12 \%$ de la muestra calificó dentro de la categoría de síntomas de probable episodio depresivo mayor (EDM) (13.3\% D.F. y 9.2\% Michoacán). La proporción fue significativamente mayor en las mujeres $\left(\chi^{2}=56.294\right.$, $\mathrm{gl}=2, \mathrm{p}<.001$ ). Los estudiantes de la Ciudad de México tuvieron una proporción significativamente mayor de síntomas de probable EDM que los estudiantes de Michoacán ( $\left.\chi^{2}=30.78, g l=2, p<.001\right)$.

\section{Discusión y conclusión}

Dada la proporción de adolescentes que presentaron síntomas clínicamente significativos, es necesario crear acciones de información, sensibilización y capacitación para padres, educadores, profesionales de la salud y adolescentes en cuanto a la relevancia de atender la depresión y mejorar el acceso a los servicios de atención especializada.

La CESD-R puede ser una alternativa rápida para la detección oportuna del probable episodio depresivo mayor, pero faltaría construir el mecanismo para derivar a los individuos en riesgo a los servicios de salud mental pertinentes, así como estrategias para garantizar que éstos sean de calidad.

Palabras clave: Adolescentes, estudiantes, depresión, CESD-R.

Dirección de Investigaciones Epidemiológicas y Psicosociales, Instituto Nacional de Psiquiatría Ramón de la Fuente Muñiz, México.

2 Department of Public Health Analysis. Morgan State University, USA.

Facultad de Psicología. Universidad Michoacana de San Nicolás de Hidalgo, Morelia, Mich., México.

Correspondencia: Dra. María Elena Rivera Heredia, profesora investigadora de la Facultad de Psicología, Universidad Michoacana de San Nicolás de Hidalgo. Gral. Francisco Villa 450, Edificio A, colonia doctor Miguel Silva. 58120, Morelia, Mich. Tel: 01(443) 317 - 8951 y 312 - 9909 , ext. 126. Fax: 01 (443) 312 - 9913. E-mail: maelenarivera@gmail.com

Recibido: 19 de febrero de 2014. Aceptado: 27 de junio de 2014 


\section{ANTECEDENTES}

La depresión es un trastorno con una prevalencia cada vez mayor entre la población adolescente..$^{1-3}$ Los procesos propios de esta etapa pueden incrementar la probabilidad de que los individuos se expongan a situaciones difíciles de afrontar que resulten estresantes. Así, es necesario generar enfoques conceptuales que tengan una aplicación práctica y que permitan distinguir entre las expresiones afectivas, cognitivas, somáticas y conductuales normales durante este periodo de aquellas que se asociarían con un malestar de consecuencias negativas para la salud mental y emocional a corto y mediano plazos. ${ }^{4,5}$

Existe evidencia que indica que el trastorno depresivo mayor y la distimia en niños y adolescentes implican un riesgo elevado de que se presenten episodios depresivos recurrentes, asociados con suicidios y con otros comportamientos autodestructivos que se pueden prolongar hasta la edad adulta. ${ }^{6,7}$ También se sabe que la depresión se relaciona con diversos factores que dificultan su atención, que empeoran su pronóstico y que aumentan el riesgo de otras conductas problemáticas. ${ }^{8}$ Entre ellos están el nivel socioeconómico, ${ }^{9}$ la historia familiar con problemas de depresión y de consumo de alcohol, ${ }^{10,11}$ las experiencias con la violencia, ${ }^{12,13}$ el abuso físico o sexual ${ }^{14}$ y el consumo de tabaco y de drogas ilegales. ${ }^{15-17}$

La presencia de sintomatología depresiva durante la adolescencia es un importante indicador de una vulnerabilidad del estado emocional, cuya gravedad puede afectar diversas áreas de la vida y aumentar la probabilidad de comorbilidad con otros trastornos psiquiátricos. ${ }^{1,18-20}$ Aunque es necesaria su detección oportuna, ésta se complica dentro de los sistemas educativos y de salud de México y otros países debido a insuficiencias de infraestructura y recursos humanos. ${ }^{21}$ Esta situación representa un reto, dadas la magnitud y la creciente tendencia de la depresión entre la población adolescente. Todo ello vuelve prioritario dirigir esfuerzos para su detección temprana a fin de proporcionar una atención oportuna y crear estrategias para su prevención, además de promover el desarrollo psicosocial de los adolescentes.

En este trabajo se describe la presencia de síntomas depresivos en adolescentes de la Ciudad de México y del Estado de Michoacán. Se presenta asimismo una comparación por sexo y entidad federativa a partir de las categorías clínicas generadas con la versión revisada de la CES-D (CESD-R).

\section{MÉTODO}

Se hicieron dos estudios transversales con muestras no probabilísticas de la Ciudad de México y del Estado de Michoacán.

Ciudad de México. Los datos se recabaron con todos los estudiantes inscritos en dos escuelas de educación media, ubicadas en el Centro Histórico de la Ciudad de México. Los planteles escolares participantes se seleccionaron mediante un criterio de contraste con base en el rendimiento académico a partir de indicadores de la Secretaría de Educación Pública. El primero tenía el mayor índice de aprobación académica y el otro, el de mayor reprobación. ${ }^{19,22,23}$

Michoacán. La información se recabó con estudiantes del primer año de secundaria de instituciones educativas públicas de poblaciones de cada una de las diez regiones socioeconómicas del Estado de Michoacán: 1. Zamora, de la región de Lerma-Chapala; 2. Zacapu, de la región del Bajío; 3. Morelia, de la región de Cuitzeo; 4. Ciudad Hidalgo, de la región de Oriente; 5 . Apatzingán, de la región de Tepalcatepec; 6. Uruapan, de la región Purépecha; 7. Pátzcuaro, de la región de Pátzcuaro-Zirahuén; 8. Huetamo, de la región de Tierra Caliente; 9. Lázaro Cárdenas, de la región de Sierra Costa, y 10. Ario de Rosales, de la región de Infiernillo. Los planteles escolares participantes se seleccionaron por cuota (un grupo de cada plantel por cada región participante).

\section{Participantes}

Ciudad de México. La muestra fue de 1549 estudiantes (54\% hombres, $46 \%$ mujeres), con una media de edad de 14 años (desviación estándar, $\mathrm{DE}=1.2$ ); $83 \%$ informaron ser estudiantes de tiempo completo la mayor parte del año anterior al estudio y que no trabajaban recibiendo un sueldo (84\%). La mayoría (81\%) tenía padre o figura paterna y 95\% tenía madre o figura materna.

Michoacán. La muestra fue de 578 estudiantes (47.9\% hombres, $52.1 \%$ mujeres), con una media de edad de 13.5 años ( $\mathrm{DE}=0.7) ; 34.4 \%$ de ellos tenía padre migrante, $13.1 \%$ tenía madre migrante y $17.2 \%$ tenía por lo menos un hermano migrante.

\section{Instrumento}

En las dos muestras se incluyó la CESD-R en cuestionarios con otras escalas, ya que formó parte de proyectos de in-

Cuadro 1. Criterios para detección con la CESD-R y para diagnóstico clínico

\begin{tabular}{|c|c|c|}
\hline Categorías del CESD-R & Criterios del CESD-R & Criterios clínicos \\
\hline $\begin{array}{l}\text { Síntomas clínicamente significati- } \\
\text { vos de probable EDM }\end{array}$ & $\begin{array}{l}\text { Existencia de síntomas en, por lo menos, cinco di- } \\
\text { mensiones que incluyan la presencia de anhedonia } \\
\text { o disforia durante dos semanas. }\end{array}$ & $\begin{array}{l}\text { Presencia de cinco síntomas clínicos de depresión, } \\
\text { inclusive anhedonia o disforia durante dos sema- } \\
\text { nas. }\end{array}$ \\
\hline $\begin{array}{l}\text { Probable episodio depresivo sub- } \\
\text { umbral }\end{array}$ & $\begin{array}{l}\text { Calificación de } 16 \text { puntos (media más una } \mathrm{DE} \text { ) } \circ \\
\text { más y no pertenecer a la categoría anterior. }\end{array}$ & $\begin{array}{l}\text { Presencia de síntomas clínicos de depresión, exclu- } \\
\text { yendo anhedonia o disforia. }\end{array}$ \\
\hline $\begin{array}{l}\text { Sin síntomas clínicamente signifi- } \\
\text { cativos de probable EDM }\end{array}$ & $\begin{array}{l}\text { Incluye a quienes calificaron por debajo de } 16 \text { en } \\
\text { la versión revisada. }\end{array}$ & Ausencia de síntomas clínicos de depresión. \\
\hline
\end{tabular}


Cuadro 2. Proporción de estudiantes que calificaron en las categorías de la CESD-R, por sexo

\begin{tabular}{|c|c|c|c|c|c|c|}
\hline \multirow{2}{*}{ Categorías del CESD-R } & \multicolumn{2}{|c|}{ Total } & \multicolumn{2}{|c|}{ Hombres } & \multicolumn{2}{|c|}{ Mujeres } \\
\hline & $\mathrm{F}$ & $\%$ & $\mathrm{~F}$ & $\%$ & $\mathrm{~F}$ & $\%$ \\
\hline $\begin{array}{l}\text { Síntomas clínicamente } \\
\text { significativos de proba- } \\
\text { ble EDM }\end{array}$ & 304 & 12.4 & 101 & 7.7 & 200 & $17.8^{*}$ \\
\hline $\begin{array}{l}\text { Probable episodio de- } \\
\text { presivo subumbral }\end{array}$ & 667 & 27.3 & 372 & 28.5 & 292 & 25.9 \\
\hline $\begin{array}{l}\text { Sin síntomas clínica- } \\
\text { mente significativos de } \\
\text { probable EDM }\end{array}$ & 1473 & 60.3 & 834 & 63.8 & 634 & 56.3 \\
\hline
\end{tabular}

vestigación más amplios. La Escala de Depresión del Centro de Estudios Epidemiológicos (CES-D) ${ }^{24}$ es un instrumento de tamizaje válido y confiable que proporciona datos para detectar sintomatología depresiva. Sin embargo, y dado el carácter dinámico del fenómeno y de las poblaciones, se hizo una actualización de la misma para que la medición concordara con los criterios diagnósticos de Episodio de Depresión Mayor (EDM) del DSM-IV ${ }^{25}$ (cuadro 1), lo que generó la versión revisada CESD-R, ${ }^{26,27}$ que tiene características psicométricas satisfactorias en poblaciones mexicanas. ${ }^{27,28}$

\section{Procedimiento y consideraciones éticas}

Se estableció contacto con autoridades de la SEP y de los planteles para obtener los permisos correspondientes. Los participantes recibieron un formato escrito de consentimiento informado en que se explicaban las condiciones de su inclusión en el estudio, que su participación era voluntaria y que el registro de la información era anónimo. La recolección de datos se efectuó en los salones de clase con el instrumento en formato autoaplicable de lápiz-papel. No se presentaron rechazos. La aplicación la realizó un equipo de psicólogos en formación, previo curso de capacitación y entrenamiento y sin la presencia de los maestros ni de otra autoridad escolar. Para capturar los datos, se utilizó la versión 15.0 de SPSS $^{29}$ y, para el análisis y procesamiento de los mismos, se utilizó el programa STATA11.0. ${ }^{30}$

\section{RESULTADOS}

De los 2127 estudiantes de la muestra, 12\% calificaron con un puntaje que los ubicó dentro de la categoría de síntomas clínicamente significativos de un probable episodio depresivo mayor (EDM); de éstos, la proporción fue significativamente mayor entre las mujeres $\left(\chi^{2}=56.294, \mathrm{gl}=2, \mathrm{p}<.001\right)$ (cuadro 2).

Los estudiantes de la Ciudad de México obtuvieron una proporción significativamente más elevada de casos dentro de la categoría de síntomas clínicamente significativos de un probable EDM que los del Estado de Michoacán. Las comparaciones entre hombres y mujeres mostraron que, en ambos casos, los estudiantes de la Ciudad de México presentaron proporciones significativamente mayores de síntomas de un probable EDM (cuadro 3).

La comparación por edad entre los adolescentes de ambas entidades sólo mostró diferencias significativas en el grupo de 13 años y menores (cuadro 4).

\section{DISCUSIÓN Y CONCLUSIÓN}

En virtud de las categorías diagnósticas que presenta la CESD-R y de la concordancia de éstas con los criterios clínicos para el diagnóstico de los trastornos afectivos propuestos por el DSM-IV, 9.2\% de la muestra de Michoacán y $13.3 \%$ de la del D.F. se ubicaron dentro del rango de un probable EDM, situación que amerita atención médica y psicológica dados los efectos que tiene este trastorno a lo largo de la vida si no se recibe la atención oportuna y apropiada. ${ }^{18-20,31}$

Dado que $12.4 \%$ de los adolescentes de ambas muestras presentaron síntomas clínicamente significativos para un probable EDM, cabe reflexionar sobre la suficiencia de la infraestructura física y humana necesaria para brindar atención adecuada a estos jóvenes, ${ }^{21}$ tanto en las diez regiones de Michoacán como en la Ciudad de México. Asimismo, se subraya la necesidad de crear acciones de información, sensibilización y capacitación para padres, educadores, profesionales de la salud y adolescentes en cuanto a la relevancia

Cuadro 3. Proporción de estudiantes que calificaron en las categorías de la CESD-R, por sexo y estado

\begin{tabular}{|c|c|c|c|c|c|c|c|c|c|c|c|c|}
\hline \multirow[b]{3}{*}{ Categorías del CESD-R } & \multicolumn{6}{|c|}{ Estudiantes D.F. } & \multicolumn{6}{|c|}{ Estudiantes Michoacán } \\
\hline & \multicolumn{2}{|c|}{ Hombres } & \multicolumn{2}{|c|}{ Mujeres } & \multicolumn{2}{|c|}{ Total } & \multicolumn{2}{|c|}{ Hombres } & \multicolumn{2}{|c|}{ Mujeres } & \multicolumn{2}{|c|}{ Total } \\
\hline & $\mathrm{F}$ & $\%$ & $\mathrm{~F}$ & $\%$ & $\mathrm{~F}$ & $\%$ & $\mathrm{~F}$ & $\%$ & $\mathrm{~F}$ & $\%$ & $\mathrm{~F}$ & $\%$ \\
\hline $\begin{array}{l}\text { Síntomas clínicamente signifi- } \\
\text { cativos de probable EDM }\end{array}$ & 88 & 8.5 & 161 & 19.4 & 249 & 13.3 & 13 & 4.8 & 39 & 13.2 & 52 & 9.2 \\
\hline $\begin{array}{l}\text { Probable episodio depresivo } \\
\text { subumbral }\end{array}$ & 321 & 31.0 & 226 & 27.2 & 547 & 29.3 & 51 & 18.8 & 66 & 22.4 & 117 & 20.6 \\
\hline $\begin{array}{l}\text { Sin síntomas clínicamente sig- } \\
\text { nificativos de probable EDM }\end{array}$ & 626 & 60.5 & 444 & 53.4 & 1070 & 57.3 & 208 & 76.5 & 190 & 64.4 & 398 & 70.2 \\
\hline
\end{tabular}

Comparación entre estudiantes del D.F. y Michoacán: $\chi^{2}=30.78, \mathrm{gl}=2, \mathrm{p}<.001$.

Comparación entre hombres del D.F. y hombres de Michoacán: $\chi^{2}=23.87, \mathrm{gl}=2, \mathrm{p}<.001$.

Comparación entre mujeres del D.F. y mujeres de Michoacán $\chi^{2}=11.25, g l=2, p=.004$. 
Jiménez Tapia et al.

Cuadro 4. Proporción de estudiantes que calificaron en las categorías de la CESD-R, por edad y estado

\begin{tabular}{|c|c|c|c|c|c|c|c|c|c|c|c|c|}
\hline \multirow[b]{3}{*}{ Categorías del CESD-R } & \multicolumn{6}{|c|}{ Estudiantes D.F. } & \multicolumn{6}{|c|}{ Estudiantes Michoacán } \\
\hline & \multicolumn{2}{|c|}{$\leq 13$} & \multicolumn{2}{|c|}{14} & \multicolumn{2}{|c|}{$\geq 15$} & \multicolumn{2}{|c|}{$\leq 13$} & \multicolumn{2}{|c|}{14} & \multicolumn{2}{|c|}{$\geq 15$} \\
\hline & $\mathrm{F}$ & $\%$ & $\mathrm{~F}$ & $\%$ & $\mathrm{~F}$ & $\%$ & $\mathrm{~F}$ & $\%$ & $\mathrm{~F}$ & $\%$ & $\mathrm{~F}$ & $\%$ \\
\hline $\begin{array}{l}\text { Síntomas clínicamente signifi- } \\
\text { cativos de probable EDM }\end{array}$ & 107 & 12.5 & 77 & 12.7 & 68 & 16.4 & 20 & 7.1 & 27 & 10.9 & 5 & 13.5 \\
\hline $\begin{array}{l}\text { Probable episodio depresivo } \\
\text { subumbral }\end{array}$ & 279 & 32.6 & 162 & 26.8 & 109 & 26.3 & 58 & 20.5 & 50 & 20.2 & 9 & 24.3 \\
\hline $\begin{array}{l}\text { Sin síntomas clínicamente sig- } \\
\text { nificativos de probable EDM }\end{array}$ & 471 & 55.0 & 366 & 60.5 & 237 & 57.2 & 205 & 72.4 & 171 & 69.0 & 23 & 62.2 \\
\hline
\end{tabular}

de atender el fenómeno de la depresión, así como de analizar la distribución y el acceso de la población a los servicios de atención especializada.

Los resultados de este estudio concuerdan con la evidencia en cuanto a que un mayor porcentaje de mujeres presentan sintomatología depresiva., ${ }^{1,32}$ Persiste, sin embargo, la necesidad de revisar la sensibilidad de la escala por sexo y considerar la posibilidad de buscar reactivos diseñados para captar las expresiones de síntomas depresivos más específicas de la población masculina.

Los adolescentes de la muestra del Distrito Federal presentaron una proporción significativamente más elevada de casos de síntomas clínicamente significativos de un probable EDM que los de Michoacán. Es posible que estos últimos pudieran estar expuestos a menos factores de estrés dado que residen en comunidades más pequeñas, con mayor interacción social entre sus miembros y mayor contacto con la naturaleza. Se debe mencionar que la aplicación se realizó en fechas anteriores a diversos disturbios sociales, relacionados con situaciones de violencia e inseguridad en el marco de la lucha contra el narcotráfico, que ocurrieron en diciembre del 2010. Es probable que los niveles de depresión encontrados sean superiores en el momento actual, ya que se sabe que la violencia incrementa la vulnerabilidad ante la depresión y otros problemas de salud mental,12,13,33 por lo que se abre la necesidad de efectuar nuevas mediciones en esta población. Además, habría que considerar que los adolescentes que participaron en este estudio eran alumnos activos de escuelas secundarias al momento de la aplicación, lo que representaría un factor de protección. Por lo anterior, es posible que los jóvenes de la misma edad que no asistan a la escuela puedan tener una mayor proporción de síntomas clínicos de depresión, lo que a su vez representaría una futura línea de investigación. Asimismo, aunque la CESD-R puede ser una alternativa rápida y ágil para la detección oportuna del episodio depresivo mayor, ${ }^{28}$ aún falta construir el mecanismo que sirva para derivar a los individuos en riesgo a los servicios de salud pertinentes, así como estrategias para garantizar que estos servicios sean eficientes y de calidad.

Este trabajo presenta algunas aportaciones importantes: 1. los datos se obtuvieron de un sector de la población que, por su etapa vital, es particularmente vulnerable a factores de riesgo que impactan en su estado de ánimo; 2. se utilizó la versión revisada del CES-D, cuya vinculación con los criterios diagnósticos del DSM-IV permite construir un lenguaje común para los profesionales de la salud; 3. se describe la proporción de jóvenes que presentan un probable EDM en una metrópoli y en un Estado del interior del país, y 4. plantea la necesidad de contar con una mayor infraestructura física y humana, así como de una mayor capacitación para tratar los trastornos del estado de ánimo en médicos y psicólogos.

Con todo, el trabajo también deja algunas áreas por refinar en estudios posteriores. Así, por ejemplo, habría que desarrollar estudios con muestras probabilísticas representativas y considerar la inclusión de adolescentes en diferentes circunstancias escolares (estudiantes regulares y no regulares).

\section{Financiamiento}

Ninguno.

\section{Conflicto de intereses}

Los autores declararon no tener conflicto de intereses.

\section{Agradecimientos}

Al programa de mejoramiento al profesorado PROMEP-UMSNH252 por su apoyo al proyecto de investigación "Promoción de la salud y prevención de conductas problema en estudiantes de secundaria", así como a Lizeth Guadalupe Martínez Servín, becaria de este proyecto.

\section{REFERENCIAS}

1. Birmaher B, Ryan ND, Williamson DE, Brent DA et al. Childhood and adolescent depression, I: A review of the past 10 years. J Am Acad Child Adolsc Psychiatry 1996;35:1427-1439.

2. Benjet C, Borges G, Medina-Mora ME, Fleiz C et al. La depresión con inicio temprano: Prevalencia, curso natural y latencia para buscar tratamiento. Salud Pública Mex 2004;46(5):417-423.

3. Benjet C, Borges G, Medina-Mora ME, Méndez E et al. Diferencias de sexo en la prevalencia y severidad de trastornos psiquiátricos en adolescentes de la Ciudad de México. Salud Mental 2009;31:155-163.

4. De la Fuente R, Medina-Mora ME, Caraveo J. Salud mental en México. México: Fondo de Cultura Económica; 1997. 
5. Weller EB, Weller RA. Depression in adolescents: growing pains or true morbidity. J Affect Disord 61 2000:(Suppl 1):9-13.

6. Weisman M, Wolf S, Golsdatein R. Depressed adolescents grown up. JAMA 1999; 218(18):1707-1713.

7. Giaconia R, Reinherz H, Paradis A, Carmola-Hauf A et al. Major depression and drug disorders in adolescence: general and specific impairments in early adulthood. J Am Acad Child Adolesc Psychiatry 2001;40(12):1426-1433.

8. Wu P, Hoven C, Okezie N, Fuller C et al. Alcohol abuse and depression in children and adolescents. J Child Adolesc Subst Abuse 2007;17(2):51-69.

9. Hasin D, Goodwin R, Stinson F, Grant B. Epidemiology of major depressive disorder: Results from the national epidemiologic survey on alcoholism and related conditions. Arch Gen Psychiatry 2005;62(10):1097-1106.

10. Anda R, Whitfield L, Felitti V, Chapman D et al. Adverse childhood experiences, alcoholic parents, and later risk of alcoholism and depression. Psychiatr Serv 2002;53(8):1001-1009.

11. Olfson M, Marcus S, Druss B, Alan-Pincus $\mathrm{H}$ et al. Parental depression, child mental health problems, and health care utilization. Med Care 2003;41(6):716-721.

12. Reinherz H, Paradis A, Giaconia R, Stashwick C et al. Childhood and adolescent predictors of major depression in the transition to adulthood. Am J Psychiatry 2003;160(12):2141-2147.

13. Castillo-Manzano R, Arankowsky-Sandoval G. Violencia intrafamiliar como factor de riesgo para trastorno depresivo mayor en mujeres: estudio de casos y controles. Rev Biomédica 2008;19(3):128-133.

14. Chapman D, Whitfield C, Felitti V, Dube S et al. Adverse childhood experiences and the risk of depressive disorders in adulthood. J Affect Disord 2004;82(2):217-225.

15. Martini S, Wagner F, Anthony J. The association of tobacco smoking and depression in adolescence: evidence from the United States. Subst Use Misuse 2002;37(14):1853-1867.

16. Lenz B. Tobacco, depression, and lifestyle choices in the pivotal early college years. J Am Coll Health 2004;52(5):213-220.

17. Milani R, Parrott A, Turner J, Fox H. Gender differences in self-reported anxiety, depression, and somatization among ecstay/MDMA polydrug users, alcohol/tobacco users, and nondrug users. Addic Behav 2004;29:965-971.

18. Rivera-Heredia ME, Andrade P. (2006). Recursos individuales y familiares que protegen al adolescente del intento suicida. Rev Intercont Psicol Educ 2006;8(2):23-40.

19. Jiménez JA, Mondragón L, González-Forteza C. Self-esteem, depressive symptomatology, and suicidal ideation in adolescents: Results of three studies. Salud Mental 2007;30(5):20-26.
20. Pérez-Amezcua B, Rivera-Rivera L, Atienzo E, De Castro F et al. Prevalencia y factores asociados a la ideación e intento suicida en adolescentes de educación media superior de la República mexicana. Salud Pública Mex 2010;52(4):324-333.

21. Benjet C, Borges G, Medina-Mora ME, Zambrano J et al. Youth mental health in a populous city of the developing world: results from the Mexican adolescent mental health survey. J Child Psychol Psychiatry 2009;50(4):386-395.

22. González-Forteza C, Ramos-Lira L, Caballero-Gutiérrez MA, Wagner FA. Correlatos psicosociales de depresión, ideación e intento suicida en adolescentes mexicanos. Psicothema 2003;15(4):524-532.

23. Ramos-Lira L, Gonzalez-Forteza C, Wagner FA. Violent victimization and drug involvement among Mexican middle school students. Addiction 2006;101(6):850-856.

24. Radloff L. The CES-D Scale: A self report depression scale for research in the general population. Appl Psychological Measurement 1977;1:385-401.

25. American Psychiatric Association. DSM-IV. Barcelona, España: MASSON; 1997.

26. Eaton W, Muntaner C, Smith C. Revision of the Center of Epidemiological Studies Depression (CES-D) Scale. Baltimore: Johns Hopkins University Prevention Center; 1998.

27. Reyes M, Soto A, Milla J, García-Vázquez A et al. Actualización de la escala de depresión del Centro de Estudios Epidemiológicos (CESD). Estudio piloto en una muestra geriátrica mexicana. Salud Mental 2003;26(1):59-68.

28. González-Forteza C, Jiménez JA, Ramos-Lira L, Wagner F. Aplicación de la escala de depresión del Center of Epidemiological Studies en adolescentes de la Ciudad de México. Salud Pública Mex 2008;50(4):292-299.

29. SPSS para Windows, versión 15.0. Illinois: SPSS INC; 2006.

30. Stata Statistical Software: Release 11. College Station. Texas: Stata Corp; 2010.

31. Birhamer B, Williamson D, Dahl R, Axelson D et al. Clinical presentation and course of depression in youth: Does onset in childhood differ from onset in adolescence? J Am Acad Child Adolesc Psychiatry 2004;43(1):63-70.

31. Osorno-Munguía JR, Vallejo-Casarín A, Segura B, Mazadiego TJ. Evaluación del funcionamiento diferencial de los ítems (DIF) de la escala de depresión del Centro para Estudios Epidemiológicos (CES-D) respecto del género en una muestra de adolescentes mexicanos. Revisa Electrónica Psicología Iztacala 2008;11(2):138-152.

33. Borges G, Medina-Mora ME, Orozco R, Ouéda C et al. Distribución y determinantes sociodemográficos de la conducta suicida en México. Salud Mental 2009;32:413-425. 